\title{
Taking a Load Off Diagnosing Constipation: Utility of the Plain Film
}

\author{
Vikram Rangan ${ }^{1}$
}

Published online: 9 October 2019

(c) Springer Science+Business Media, LLC, part of Springer Nature 2019

Constipation is a common complaint of patients cared for in gastroenterology and primary care clinics. Functional constipation, diagnosed clinically, is often associated with diagnostic uncertainty, since patients frequently describe alternating constipation and diarrhea. Indeed, fluctuating bowel habits led to many irritable bowel syndrome (IBS) patients being subtyped as IBS-U (rather than IBS-C, IBSD, or IBS-M) under the previously utilized Rome III diagnostic criteria [1]. This difficulty in making a clinical diagnosis of constipation can lead to challenges in determining appropriate medical management, for example, a mixture of constipation and diarrhea symptoms could represent either constipation with overflow diarrhea or the mixed subtype of irritable bowel syndrome. A laxative may be helpful for both symptoms in the former case, but may worsen underlying diarrhea in the latter case.

An abdominal X-ray is one of the few diagnostic modalities that can in theory objectively quantify fecal burden, and either corroborate or refute a clinical diagnosis of constipation in cases of diagnostic uncertainty. Thus far, however, there has been a lack of evidence of the utility of radiographic fecal burden measurement (estimation of the amount of fecal material in the colonic lumen) in adult patients. As the authors note, much of the existing literature regarding radiographically documented fecal loading has been in the pediatric population, and even in this population, there is a lack of clear evidence of its utility. Given this lack of evidence (particularly in adult patients), and given the non-trivial ionizing radiation dose associated with abdominal plain film [2], further study of its potential role in adult patients is clearly needed.

In this issue of Digestive Diseases and Sciences, Pleasant et al. [3] reported a retrospective study of 319 adult patients (median age 57.9 years, and predominantly female)

\footnotetext{
Vikram Rangan

vrangan@bidmc.harvard.edu
}

1 Division of Gastroenterology, Department of Medicine, Beth Israel Deaconess Medical Center, Harvard Medical School, 330 Brookline Avenue, Dana 535, Boston, MA 02215, USA who were seen by a gastroenterologist or urogynecologist at the University of Michigan between 2005 and 2017, and who underwent an abdominal X-ray for assessment of fecal loading. The authors investigated the association between radiographic fecal burden (which was determined on the basis of "fecal loading" or "fecal matter" in the radiology report) and clinically reported patient symptoms, focusing on clinical symptoms of constipation, diarrhea, bloating, and accidental bowel leakage. The authors distinguished between cases in which the above symptoms were noted as the chief complaint, versus as a listed symptom. The vast majority of these 319 patients $(84.0 \%$ ) had radiographic documentation of fecal loading. Key findings were that a chief complaint of constipation was an independent predictor of having fecal loading on multiple logistic regression analysis, whereas chief complaints of "accidental bowel leakage" or "other" were not independent predictors of fecal loading (those with chief complaint of diarrhea served as the reference group in this analysis). Constipation was not an independent predictor of fecal loading when listed as a symptom rather than as a chief complaint. Among other listed symptoms, bloating was the only one noted to be an independent predictor of fecal loading. Among demographic factors, BMI was associated with fecal loading, with each one unit increase in BMI associated with an $8 \%$ increase in the odds of radiographic fecal loading.

This study reported data exclusively gathered on adult outpatients and did not utilize a formal scoring system to assess fecal burden. The selection of outpatients who reported GI symptoms was a unique aspect of this study compared to other adult studies performed in the emergency room setting [4]. Within this narrower population, the authors did indeed find clinical utility to this test, specifically noting that constipated patients were significantly more likely to have fecal loading compared with diarrhea patients, and that a complaint of bloating was also associated with fecal loading. Although constipation remains a clinical rather than radiographic diagnosis, abdominal X-ray can nonetheless serve as a helpful additional data point in clarifying the clinical picture in cases of uncertainty. As the first 
study to demonstrate a relationship between radiographic fecal burden and clinically reported patient symptoms in an adult outpatient population, this study does clearly advance clinical practice.

Despite the demonstrated association between radiographic fecal burden and clinical constipation and bloating symptoms, the exact clinical implication of a radiographic finding of fecal loading still needs to be interpreted cautiously, and on a case-by-case basis. As the authors note, fecal loading was a very common finding, noted in $84 \%$ of individuals in this study (including $80 \%$ of those whose chief complaint was something other than constipation, and $>70 \%$ of those whose chief complaint was diarrhea), with the authors specifically noting that moderate fecal loading in multiple bowel compartments was most commonly noted in the radiographic report. Fecal loading can be seen both in those with and without GI symptoms [5], indicating that the initial symptoms cannot definitively be tied to fecal loading in many cases. Bloating in particular is a multifactorial symptom with many potential causes [6], requiring careful consideration of all relevant clinical data before attributing this symptom to constipation based on the results of abdominal X-ray.

The authors noted that those with diarrhea and bowel leakage were no more likely to have fecal loading than those without these symptoms. In their discussion, the authors noted that this study did not distinguish between diarrhea as a global symptom and diarrhea suspected to be secondary to overflow from constipation. There were likely both types of diarrhea affecting the patients in this study, with a resulting wide variety of fecal loading. Thus, the lack of association between diarrhea and fecal loading does not diminish the utility of the abdominal X-ray in identifying cases of diarrhea or bowel leakage due to constipation with overflow. Nevertheless, it does point to the importance of performing this test only for selected indications, most notably, when constipation with overflow is suspected to be the underlying cause of a given patient's symptoms based on history taking. In this setting, the presence of fecal loading would serve as a pertinent data point supporting this clinical suspicion (while the absence of fecal loading would argue strongly against it). By contrast, abdominal X-ray ordered routinely for the evaluation of diarrhea or IBS-M is likely to be much less informative (particularly given that in the latter condition, fecal load may significantly vary on a day-to-day basis).

Among the key challenges, preventing the increased use of abdominal X-ray to assess fecal loading (alluded to and discussed by the authors as well) is variability in interpretation and reporting. Radiologists may not routinely comment on fecal burden on abdominal plain film unless noted to be significantly abnormal. Uniform usage of a standardized scoring system to assess fecal burden would seemingly be helpful in addressing this challenge. There are three commonly cited scoring systems for assessing fecal burden in children referenced by this paper. One is the system described by Leech et al. [7] that entails demarcating the colon into three segments (right colon, left colon, and rectosigmoid colon) using anatomic landmarks on plain film, and scoring fecal burden on a $0-5$ scale in each segment. This system correlates with the Bristol stool form, colonic transit time, and subjective GI symptoms in children [8]. In a recent study applying this scoring system to adults, it was associated with slow transit constipation based on radiopaque marker testing [9], though of note, a prior study of this scoring system showed no association with clinical symptoms [10].

Widespread adoption will likely be a challenge, and indeed the authors argue that the measurement of fecal burden in this study (which did not utilize a standardized scoring system) may be more generalizable to current clinical practice. While this assumption is likely correct, the lack of uniformity in reporting of fecal loading without a standardized scoring system is reflected by the authors' observation that severity of fecal loading could not be clearly categorized in $>25 \%$ of those with fecal loading in this study (in $10.8 \%$ of those with fecal loading, severity was not specified, and in $17.2 \%$, it was classified as "other," rather than mild/moderate/severe). Similarly of 153 patients in whom fecal loading was noted in multiple compartments, over 40\% (63/153) did not have documentation of which compartments were involved, speaking to the lack of standardization in documentation. In the future, it may be informative to retrospectively review these images with a trained radiologist and assign a score according to published criteria. This would not only control for heterogeneity in the interpretation of these studies, but also allow for exploration of whether there is a severity cutoff (rather than the binary presence or absence of fecal loading) that more clearly identifies those with constipation.

Ultimately, this noteworthy study has demonstrated for the first time an association between radiographic fecal loading and clinically reported constipation. While abdominal X-ray alone cannot be used to make a clinical diagnosis, and while the finding of fecal loading must be interpreted cautiously, this study suggests that abdominal radiography remains of importance in the assessment and management of selected GI complaints.

\section{Compliance with Ethical Standards}

Conflict of interest The author declares that they have no conflict of interest 


\section{References}

1. Simren M, Palsson OS, Whitehead WE. Update on Rome IV criteria for colorectal disorders: Implications for clinical practice. Curr Gastroenterol Rep. 2017;19:15.

2. Mettler FA, Huda W, Yoshizumi TT, Mahesh M. Effective doses in radiology and diagnostic nuclear medicine: A catalog. Radiology. 2008;248:254-263.

3. Pleasant V, Sammarco A, Keeney-Bonthrone G, Bell S, Saad R, Berger MB. Use of X-ray to assess fecal loading in patients with gastrointestinal symptoms. Dig Dis Sci. (Epub ahead of print). https://doi.org/10.1007/s10620-019-05770-9.

4. Driver BE, Chittineni C, Kartha G, Cole JB, Klein LR, Rischall M. Utility of plain abdominal radiography in adult ED patients with suspected constipation. Am J Emerg Med. 2008;27:158377.

5. Khan O, Shankar PR, Parikh AD, et al. Radiographic stool quantification: an equivalence study of 484 symptomatic and asymptomatic subjects. Abdom Radiol (NY). 2019;44:821-827.

6. Malagelada JR, Accarino A, Azpiroz F. Bloating and abdominal distension: Old misconceptions and current knowledge. Am J Gastroenterol. 2017;112:1221-1231.
7. Leech SC, McHugh K, Sullivan PB. Evaluation of a method of assessing faecal loading on plain abdominal radiographs in children. Pediatr Radiol. 1999;29:255-258.

8. Koh H, Lee MJ, Kim MJ, Shin JI, Chung KS. Simple diagnostic approach to childhood fecal retention using the Leech score and Bristol stool form scale in medical practice. J Gastroenterol Hepatol. 2010;25:334-338.

9. Cangemi DJ, Flanagan R, Barshop K, Kuo B, Staller K. Colonic stool burden a useful surrogate for slow transit constipation as determined by a radiopaque transit study. Am J Gastroenterol. 2019;114:519-523.

10. Cowlam S, Vinayagam R, Khan U, et al. Blinded comparison of faecal loading on plain radiography versus radio-opaque marker transit studies in the assessment of constipation. Clin Radiol. 2008;63:1326-1331.

Publisher's Note Springer Nature remains neutral with regard to jurisdictional claims in published maps and institutional affiliations. 